\title{
MACRO IMPLICATIONS OF GOVERNMENT REDISTRIBUTIVE TAX-TRANSFER POLICIES
}

\author{
Leigh TESFATSION* \\ University of Southern California, Los Angeles, CA 90007, USA \\ Received May 1981, revised version received December 1981
}

\begin{abstract}
How do macro variables such as aggregate consumption, aggregate money demand, prices, and interest rates vary in response to government attempts to redistribute income across agents through selective tax-transfer policies? This question is investigated for an overlapping generations model consisting of heterogeneously endowed three-period lived agents. In the presumably most favorable case for invariance (identical log-linear utility functions), it is shown that positive interest rate macro equilibria are invariant with respect to redistributions in social security benefits if and only if all agents initially exhibit qualitatively identical liquidity preference behavior, i.e. positive money holding only in youth, only in middle-age, or never.
\end{abstract}

\section{Introduction}

It is well known [Green (1977)] that an assumption of identical homothetic preferences in standard Walrasian general equilibrium contexts is essentially both necessary and sufficient for aggregate consumption to be invariant with respect to redistributions of income across agents. The present paper demonstrates that an assumption of identical homothetic preferences is not generally sufficient for such invariance results in microfoundations models incorporating the overlapping generations feature. An additional necessary assumption is the qualitative similarity of agent liquidity preference behavior prior to redistribution.

Specifically, the present paper considers a one-good pure exchange overlapping generations model consisting of a balanced budget government with tax-transfer powers, and three-period lived agents who receive endowments and pay taxes in their youth and middle age and receive social security benefits in their old age. Each generation comprises two agent types, distinguished by their endowment profile, social security benefits, and population size. Endowments take the form of perishable commodity-good

*This work was supported by the National Science Foundation under Grant No. ENG 7728432. A first draft was written during the author's 1980 visit at the University of Minnesota, and a second draft was presented at the Econometric Society Meetings, UC San Diego, on June 25, 1981. The author is grateful to V. Crawford, T. Groves, R. Starr, N. Wallace, and two anonymous referees for helpful comments. 
allocations which agents trade among themselves. Liquid assets are represented by a fixed stock of fiat money issued by government, and illiquid assets are represented by the opportunity, available only to young agents, of selling or buying a nominal quantity of two-period bonds (voluntary retirement annuities) to or from government in a net aggregate amount determined by government policy. Each young agent is assumed to maximize the utility of his lifetime consumption, with homothetic utility function $U: R_{++}^{3} \rightarrow R$ given by the log-linear specification $U\left(c^{1}, c^{2}, c^{3}\right) \equiv \log \left(c^{1}\right)$ $+\alpha \log \left(c^{2}\right)+\beta \log \left(c^{3}\right)$ for arbitrary discount values $\alpha>0$ and $\beta>0$.

Two questions are posed for this class of economies. First, what are the existence and uniqueness properties of the stationary competitive equilibria? Second, what macro equilibria correspond to these micro equilibria; and how do the individual macro equilibrium components (aggregate consumption, aggregate money holding, real bond price, and bond interest rate) vary in response to government attempts to redistribute income across agents through selective transfers in endowments and social security benefits?

A complete characterization is first obtained for the full set of stationaryprice perfect foresight competitive equilibria satisfying the usual utility maximization and market clearing conditions for money, bonds, and goods, with endogenously determined two-period bond interest rate and real bond price. The equilibria depend parametrically on the population size of each agent type, the endowments received in youth and middle age by each agent type, the utility discount values $\alpha$ and $\beta$, and the social security, tax, and net aggregate bond instruments of government. It is shown that the economies under consideration may have zero, one, two, or infinitely many equilibria, depending on the particular configuration of these parameter values, and the subset of economies with infinitely many equilibria has positive Lebesgue measure. Nonuniqueness arises even if all agents are identical. ${ }^{1}$

Implications are then drawn regarding the sensitivity of the corresponding macro equilibria to parameter variations. Particular attention is focused on distributional variations in endowments and benefits, i.e. variations in the distribution of endowments and social security benefits among agents at each time $t$ which leave aggregate endowment and benefit levels unchanged at each time $t$. The qualitative similarity/dissimilarity of agent liquidity preferences is shown to crucially affect the sensitivity of macro equilibria with respect to distributional variations.

For cxample, the real price of bonds, the bond interest rate, and the aggregate consumption profile of each generation in any positive interest rate equilibrium are shown to be invariant with respect to all local distributional variations in both endowments and social security benefits if and only if, in

\footnotetext{
${ }^{1}$ As will be clarified in section 3 , these results contrast sharply with results obtained both in standard log-linear Walrasian models and in log-linear overlapping generations models with perfect borrowing and lending.
} 
the initial equilibrium, all agents hold positive money balances only in their youth. Furthermore, the real price of bonds, the bond interest rate, and both the aggregate consumption profile and the aggregate money-holding profile of each generation in any positive interest rate equilibrium are shown to be invariant with respect to all local distributional variations in social security benefits if and only if all agents initially exhibit qualitatively identical liquidity preferences, i.e. positive money holding only in youth, only in middle-age, or never. Finally, starting in a zero interest rate equilibrium, in which all agents are indifferent between money and bonds, these latter macro variables are shown to be invariant with respect to all local distributional variations in both endowments and benefits. ${ }^{2}$

Various additional existence, uniqueness, and invariance implications are derived and graphically illustrated for the special cases of zero youth endowments, zero middle-age endowments, and zero net aggregate bonds, respectively. For example it is shown that all of the macro variables indicated above are invariant with respect to all distributional variations in youth endowments and social security benefits, both local and global, if youth endowments are positive and middle-age endowments are zero. In addition, in this case government is able to set social security benefits, taxes, and net aggregate bonds at arbitrary levels without destroying stationary equilibrium. The latter conclusion is shown to fail in the opposite case of zero youth endowments and positive middle-age endowments. These results indicate the potentially special nature of studies which postulate youth endowments only, or middle-age endowments only.

In the case of no government intervention (zero benefits, zero taxes, and zero net aggregate bonds), it is shown that infinitely many equilibria exist if youth endowments are positive, and no equilibria exist otherwise; hence government intervention is necessary for existence only in the case of zero youth endowments. Moreover, the equilibrium bond interest rate is independent of all local distributional variations in endowments only if it is zero prior to variation. Finally, if net aggregate bonds are zero, and government attempts to provide social security benefits to one agent type only, where agent types are otherwise identical, it is shown that the corresponding equilibria display a surprising degree of neutrality with respect to the particular level of benefits selected for the favored agent type.

The model is developed in section 2. Existence and uniqueness are discussed in section 3 and appendix A for the full set of competitive equilibria as a function of the basic model parameters. Distributional effects are derived and illustrated in section 4. Proof outlines are provided in appendix B.

The importance of deriving macro implications from dynamic equilibrium

\footnotetext{
${ }^{2}$ Note that the present paper only considers equilibrium aggrcgatcs of homogeneous items, i.e. quantities of the consumption good, or of dollars.
} 
models based on rational individual behavior has previously been stressed by a variety of researchers, e.g. Balasko and Shell (1981a, b), Brock and Scheinkman (1980), Cass and Shell (1980), Lucas (1980), Lucas and Sargent (1979), Sargent and Wallace (1976), and Wallace (1980a, b). However, in contrast to the present paper, most of these studies focus on the effectiveness of government monetary transfer policy for ensuring the existence of Pareto optimal outcomes in economies with perfect borrowing and lending.

\section{The model}

Consider a one-good pure exchange stationary overlapping generations model consisting of a population of heterogeneously endowed three-period lived agents and a balanced budget government with tax and transfer powers. Specifically, at each timc $t,-\infty<t<\infty$, the population consists of $N_{k}$ newly-born young agents of type $k$ endowed with $\omega_{k}^{1}$ units of the commodity good, $k=1,2$ ('the young generation'), $N_{k}$ middle-aged agents of type $k$ born at time $t-1$ and endowed at time $t$ with $\omega_{k}^{2}$ units of the commodity good, $k=1,2$, and $N_{k}$ old agents of type $k$ born at time $t-2$, $k=1,2$, who will receive no commodity endowment at time $t$ and who will die at the end of the $t$ th period $[t, t+1]$. For each $k=1,2$, it is assumed that $\omega_{k}^{i} \geqq 0$ and $\omega_{k}^{1}+\omega_{k}^{2}>0, i=1,2$. Also, the commodity good is assumed to be nonstorable.

At each time $t$ there is a pretransfer stock of fiat money held in the private sector by middle-aged and old agents of type $k$ in the form of money balances $M_{k}^{1} \geqq 0$ and $M_{k}^{2} \geqq 0$, respectively, $k=1,2$. As will be clarified below, the aggregate outstanding stock of fiat money is determined endogenously by market clearing and government budget conditions. Only social security benefits, the tax rate, and the net aggregate bond level are directly controlled by government. (The endowment redistributions considered in section 4 can be interpreted as an additional government instrument, albeit one that does not formally enter into the model.)

Let $P$ denote the fiat money price at each time $t$ of one unit of the consumption good. At each time $t$ the government levies taxes $T P \omega_{k}^{1}$ and $T P \omega_{k}^{2}$ on type $k$ young and middle-aged agents, respectively, and distributes a nominal social security payment $S_{k}$ to type $k$ old agents, where $0 \leqq T<1$ and $0 \leqq S_{k}, k=1,2$. In addition, the government stands ready to sell or purchase in net terms a certain nominal quantity $|\bar{B}|$ of two-period bonds (voluntary retirement annuities) in transaction with the young generation, $-\infty<\vec{B}<\infty$, where each bond is a promise to pay one unit of fiat money to the bearer at time $t+2$. Bonds are not privately transferable. The fiat money price of a two-period bond at each time $t$ is denoted by $P^{B}$, and the price of a two-period bond at each time $t$ in units of time $t$ consumption is denoted by $p^{B} \equiv P^{B} / P$. The two-period rate of interest on bonds at each time $t$ is thus 
given by $i \equiv\left[1-P^{B}\right] / P^{B}$. For simplicity, the model conditions will henceforth be expressed in terms of $i$ and $p^{B}$ rather than $P$ and $P^{B}$.

For each $k=1,2$ and each time $t$, the planning problem of a type $k$ young agent is assumed to be the choice of a consumption profile $c_{k} \equiv\left(c_{k}^{1}, c_{k}^{2}, c_{k}^{3}\right)$, a money holding profile $M_{k} \equiv\left(M_{k}^{1}, M_{k}^{2}\right)$, and a two-period bond purchase $\left(B_{k} \geqq 0\right)$ or sale $\left(B_{k}<0\right)$ when young, to maximize utility of consumption

$$
U\left(c_{k}^{1}, c_{k}^{2}, c_{k}^{3}\right)
$$

subject to the budget constraints

$$
\begin{aligned}
& c_{k}^{1}+(1+i) p^{B} M_{k}^{1}+p^{B} B_{k}=[1-T] \omega_{k}^{1}, \\
& c_{k}^{2}+(1+i) p^{B} M_{k}^{2}=[1-T] \omega_{k}^{2}+(1+i) p^{B} M_{k}^{1}, \\
& c_{k}^{3}=(1+i) p^{B}\left[B_{k}+S_{k}+M_{k}^{2}\right], \\
& M_{k}^{1} \geqq 0, \quad M_{k}^{2} \geqq 0,
\end{aligned}
$$

where $U: R_{+}^{3} \rightarrow R$ is defined by

$$
U\left(c^{1}, c^{2}, c^{3}\right) \equiv \log \left(c^{1}\right)+\alpha \log \left(c^{2}\right)+\beta \log \left(c^{3}\right)
$$

for arbitrary positive discount values $\alpha$ and $\beta$. Note that the planning problem (1) has no finite solution if either $p^{B}=0$ or $i<0$. If either $p^{B}=0$ or $i \leqq-1$, this is clear. If $p^{B}>0$ and $-1<i<0$, then, by setting $M_{k}^{1}-\delta$ $=M_{k}^{2} \equiv L$ and $B_{k}=-1+\varepsilon$ for some $\delta>0$ and $\varepsilon>0$ and letting $L \rightarrow+\infty$, one sees that $c_{k}^{1}$ becomes arbitrarily large while $c_{k}^{2}$ and $c_{k}^{3}$ remain constant at the positive levels $[1-T] \omega_{k}^{2}+(1+i) p^{B} \delta$ and $(1+i) p^{B}\left[\varepsilon+S_{k}\right]$, respectively. The definition of equilibrium given below will require that $p^{B}>0$ and $i \geqq 0$.

It can be shown that the solution $\left(M_{k}, B_{k}, c_{k}\right)$ for (1) is a well-defined continuous nondifferentiable function of $p^{B}$ and $i$ over $R_{++}^{2}$ (Infinitely many solutions exist for (1) when $i=0$, since agents are then indifferent between money and bonds.) The liquidity preference characteristics of this solution as a function of endowment profile are depicted in figs. 1 and 2.

As illustrated in fig. 1, the endowment space partitions into threc regions, I, II, and III, each generating solutions to (1) with distinct money-holding behavior. For example, any endowment profile $\left(\omega_{k}^{1}, \omega_{k}^{2}\right)$ in region I generates a corresponding solution to (1) in which money holding is desired only in youth, i.e. $M_{k}^{1}>0$ and $M_{k}^{2}=0$. Fig. 1 is intuitively reasonable, reflecting the desire of young agents to smooth out their endowment profiles. Fig. 1 would presumably retain its qualitative appearance even for more general utility functions. 


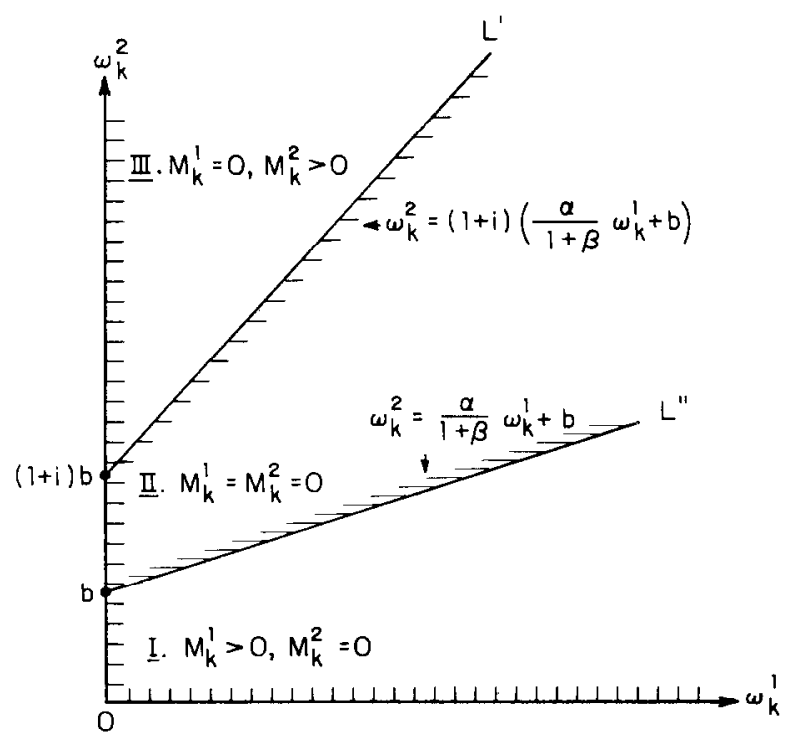

Fig. 1. Depiction of the optimal solution for problem (1) with $0<b \equiv x p^{B}[1-T]^{-1} S_{k} /(1+\beta)$, qualitatively characterizing the planned money holdings $\left(M_{k}^{1}, M_{k}^{2}\right)$ of the type $k$ young as a function of their endowments $\left(\omega_{k}^{1}, \omega_{k}^{2}\right)$ for given parameter values $\alpha, \beta, S_{k}$, and $T$, and given interes1 rate $i>0$ and real bond price $p^{B}>0$.

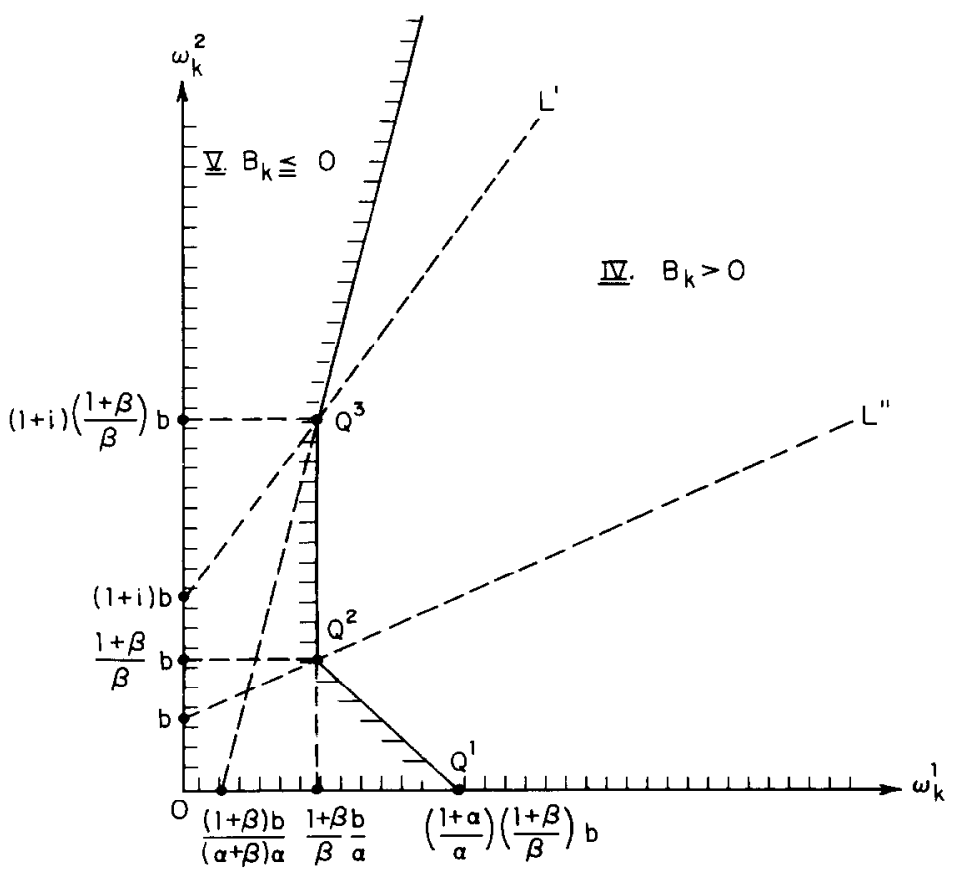

Fig. 2. Depiction of the optimal solution for problem (1) with $0<b \equiv \alpha p^{B}[1-T]^{-1} S_{k} /(1+\beta)$ and $(1+\beta) / \beta<(1+i)$, characterizing the regions IV and $\mathrm{V}$ in endowment space where the type $k$ young will seek positive and negative purchases of bonds for given parameter values $\alpha, \beta, S_{k}, T$, and given interest rate $i>0$ and real bond price $p^{B}>0$. 
Fig. 2 depicts the bond-holding behavior of young type $k$ agents as a function of endowment profile. As depicted, the endowment space partitions into two regions, IV and $\mathrm{V}$, bounded by solid lines, corresponding to solutions where bonds are bought $\left(B_{k}>0\right)$ and to solutions where bonds are sold $\left(B_{k}<0\right)$, respectively. (This bond-holding dichotomy is superimposed over the partition lines $L^{\prime}$ and $L^{\prime \prime}$ from fig. 1 in order to depict the exact correspondence of the money-holding and bond-holding partitions.) The intuitive interpretation of fig. 2 is not as totally apparent as the one for fig. 1 . Note that the bond-holding behavior of young type $k$ agents is monotonic with respect to their middle-age endowment $\omega_{k}^{2}$ for any given positive youth endowment $\omega_{k}^{1}$ if and only if their social security benefits $S_{k}$ are zero, implying $b-0 .^{3}$

Before presenting the government budget constraint and market clearing conditions for this model, certain compact notations will be introduced for describing aggregates.

\subsection{Notational conventions}

Let

$$
N \equiv\left(N_{1}, N_{2}\right), \quad \omega \equiv\left(\omega_{1}^{1}, \omega_{1}^{2}, \omega_{2}^{1}, \omega_{2}^{2}\right), \quad S \equiv\left(S_{1}, S_{2}\right)
$$

denote the vector of population sizes, endowments, and social security benefits, respectively, for agent types 1 and 2, and let

$$
\begin{aligned}
& M \equiv\left(M_{1}^{1}, M_{1}^{2}, M_{2}^{1}, M_{2}^{2}\right) \equiv\left(M_{1}, M_{2}\right), \\
& B \equiv\left(B_{1}, B_{2}\right), \\
& c \equiv\left(c_{1}^{1}, c_{1}^{2}, c_{1}^{3}, c_{2}^{1}, c_{2}^{2}, c_{2}^{3}\right) \equiv\left(c_{1}, c_{2}\right),
\end{aligned}
$$

denote the vector of money holdings, bond holdings, and consumption levels, respectively, for agent types 1 and 2 . Note, by stationarity, that aggregating endowments across a generation also yields aggregate endowments at each time $t$, and similarly for consumption, money and bond holdings, and social

\footnotetext{
${ }^{3}$ In Martins (1980) a special case of the young generation planning problem (1) is examined under the maintained hypothesis $M_{k}^{1}>0$ and $M_{k}^{2}=0$, and for parameter restrictions $\omega_{k}^{1}>0, \alpha=\beta$ $=1$, and $\omega_{k}^{2}=S_{k}=T=0$. The basic conclusion of Martins' study (1980, p. 181) is that the bond rate of interest $i$ is given by the bonds/money ratio minus one, i.e. $i=B_{k} / M_{k}^{1}-1$. Substituting Martins' parameter restrictions into (1), it can be shown that the optimal solution does satisfy this relation. However, Martins' conclusion does not generalize to the case where $\omega_{k}^{2}>0$ or $S_{k}>0$. Moreover, as figs. 1 and 2 strikingly indicate, many additional interesting features of problem (1) are obscured when one imposes the Martins restriction $\omega_{k}^{2}=S_{k}=0$.
} 
security benefits. The following notational conventions will be used to denote the indicated endowment aggregates at each time $t$ :

$$
\begin{aligned}
& N \omega \equiv N_{1}\left[\omega_{1}^{1}+\omega_{1}^{2}\right]+N_{2}\left[\omega_{2}^{1}+\omega_{2}^{2}\right] \quad \text { (aggregate endowment), } \\
& N \omega^{1} \equiv N_{1} \omega_{1}^{1}+N_{2} \omega_{2}^{1} \quad \text { (aggregate youth endowment), } \\
& N \omega^{2} \equiv N_{1} \omega_{1}^{2}+N_{2} \omega_{2}^{2} \quad \text { (aggregate middle-age endowment), } \\
& \left.N_{k} \omega_{k} \equiv N_{k}\left[\omega_{k}^{1}+\omega_{k}^{2}\right] \quad \text { (aggregate endowment of agent type } k, k=1,2\right) .
\end{aligned}
$$

Similarly, the following notational conventions will be used to denote the indicated consumption, money, bond, and social security benefit aggregates at each time $t$ :

$$
\begin{aligned}
& N c \equiv N_{1}\left[c_{1}^{1}+c_{1}^{2}+c_{1}^{3}\right]+N_{2}\left[c_{2}^{1}+c_{2}^{2}+c_{2}^{3}\right] \quad \text { (aggregate consumption), } \\
& N c^{j} \equiv N_{1} c_{1}^{j}+N_{2} c_{2}^{j}, \quad j=1,2,3 \quad \text { (aggregate age } j \text { consumption), } \\
& N M \equiv N_{1}\left[M_{1}^{1}+M_{1}^{2}\right]+N_{2}\left[M_{2}^{1}+M_{2}^{2}\right] \text { (aggregate money holdings), } \\
& N M^{j} \equiv N_{1} M_{1}^{j}+N_{2} M_{2}^{j}, \quad j=1,2 \quad \text { (aggregate age; money holdings), } \\
& N B \equiv N_{1} B_{1}+N_{2} B_{2} \quad \text { (aggregate bond holdings), } \\
& N S \equiv N_{1} S_{1}+N_{2} S_{2} \quad \text { (aggregate social security benefits). }
\end{aligned}
$$

Using these notational conventions, the government budget constraint at each time $t$ is given by

$$
p^{B} N B+T N \omega=(1+i) p^{B}[\bar{B}+N S]
$$

the goods market clearing condition at each time $t$ is given by

$$
N c=N \omega
$$

and the bond market clearing condition at each time $t$ is given by

$$
N B=\bar{B}
$$

Finally, the money market clearing condition at each time $t$ is given by

$$
(1+i) p^{B} N M+p^{B} N B+T N \omega=(1+i) p^{B}[N B+N S+N M] .
$$


Clearly (5) holds if (2) and (4) hold. Moreover, the cross-sectional budget constraints for young, middle-aged, and old agents at each time $t$ together with (3) and (4) can be shown to imply (2); hence, both (2) and (5) are superfluous conditions in the present stationary context.

Definitions will now be given for an economy and for a stationary competitive equilibrium, or equilibrium for short.

\subsection{Definitions}

A parameter vector $e=(N, \omega, \alpha, \beta, S, T, \bar{B})$ in $R^{12}$ will be called an economy if the parameter values are admissible in the sense that $N \in R_{++}^{2}, \omega \in R_{+}^{4}$ with $\omega_{k}^{1}+\omega_{k}^{2}>0, k=1,2, \alpha \in R_{++}, \beta \in R_{++}, S \in R_{+}^{2}, T \in[0,1)$, and $\bar{B} \in R$. Given any economy $e$, a vector $\left(p^{B}, i, M, B, c\right)$ in $R_{++} \times R_{+} \times R_{+}^{4} \times R^{2} \times R_{++}^{6}$ will be called an equilibrium for $e$ if $\left(M_{k}, B_{k}, c_{k}\right)$ solves the young agent planning problem (1) for the given real bond price $p^{B}$, bond interest rate $i$, and parameter values defining $e, k=1,2$, and the market clearing conditions (3) and (4) are also satisfied.

\section{Existence and uniqueness of equilibria}

Each of the theorems 3.1 through 3.8 presented in appendix A characterizes the existence and uniqueness of equilibria corresponding to a distinct mode of liquidity preference behavior for agent types 1 and 2 in terms of primitive parameter restrictions. A complete characterization is thus obtained for the stratification induced on the space of economies by the stratification of the space of competitive equilibria into distinct liquidity preference regions. Some of the complex crystalline aspects of this stratification are depicted via two-dimensional projections in the following section (figs. 3 through 6).

Theorem 3.1 establishes the nonexistence of positive interest rate equilibria with both positive youth and positive middle-age money holding, and theorem 3.2 establishes necessary and sufficient conditions for the existence of a zero interest rate equilibrium (money-bond indifference). Theorems 3.3 through 3.5 establish necessary and sufficient conditions for existence with agents exhibiting qualitatively identical liquidity preference behavior in a positive interest rate equilibrium, and theorems 3.6 through 3.8 (and their symmetrical counterparts $3.6^{*}$ through $3.8^{*}$ ) establish necessary and sufficient conditions for existence with agents exhibiting weakly and strongly dissimilar liquidity preference behavior in a positive interest rate equilibrium.

The theorems demonstrate that zero, one, two, or infinitely many equilibria may exist for an economy. As established in corollary 3.2.1, the subset of economies in $R^{12}$ with infinitely many equilibria has positive Lebesgue measure. By way of contrast, in the standard nonmonetary pure 
exchange general equilibrium model with log-linear utility functions and normalized prices the derived excess demand functions satisfy gross substitutability and indecomposability conditions which guarantee the existence of a unique equilibrium [see Nikaido (1968, pp. 305-307, 335)]. In a log-linear two-period overlapping generations model with fiat money and perfect borrowing and lending, Balasko and Shell (1981b) find that the price of money is generally indeterminate and parameterizes the monetary equilibria corresponding to any given economy, i.e. each economy has infinitely many equilibria.

One reason for this discrepancy in number of equilibria appears to be as follows. In the present model the domain of prices $P^{B}$ and $P$ in $R_{++}^{2}$ for which the excess demand correspondence maps into a nonempty subset of excess demands is neither bounded nor closed, and price normalization is not possible. The usual Kakutani fixed-point theorem argument for existence is therefore inapplicable [see Nikaido (1968, section 16)].

Further implications of theorems 3.2 through 3.8 for existence and uniqueness are discussed and illustrated in section 4 .

\section{Distributional effects}

The present section uses the theorems of appendix $A$ to explore the sensitivity of equilibria to parameter variations, with particular emphasis on variations in distribution which leave aggregate levels unchanged. In contrast to results obtained for standard log-linear Walrasian models, it is shown that macro equilibria for the present log-linear overlapping generations model are invariant with respect to redistributions in endowments and social security benefits if and only if all agents exhibit suitably similar liquidity preference behavior prior to redistribution.

The first theorem below establishes the neutrality of equilibria with respect to certain fixed-proportion variations. These results demonstrate that various normalizations could have been incorporated without loss of generality into the definition of an economy if existence had been the only question at issue.

Theorem 4.1. (Neutrality with respect to fixed-proportion variations). Let an economy $e=(N, \omega, \alpha, \beta, S, T, \bar{B})$ be given, and let $\left(p^{B}, i, M, B, c\right)$ be an equilibrium for $e$. Then the following assertions hold.

1. Money neutrality: $\left(\lambda^{-1} p^{B}, i, \lambda M, \lambda B, c\right)$ is an equilibrium for $e_{\lambda}=(N, \omega, \alpha, \beta$, $\lambda S, T, \lambda \bar{B})$ for every $\lambda>0$.

2. Endowment neutrality: $\left(\theta p^{B}, i, M, B, \theta c\right)$ is an equilibrium for $e_{\theta}=(N, \theta \omega, \alpha$, $\beta, S, T, \bar{B})$ for every $\theta>0$.

3. Population neutrality: $\left(p^{B}, i, M, B, c\right)$ is an equilibrium for $e_{\delta}=(\delta N, \omega, \alpha, \beta$, $S, T, \delta \bar{B})$ for every $\delta>0$. 
Precise definitions will now be given for distributional variation and related concepts.

\section{Definitions}

Let an economy $e=(N, \omega, \alpha, \beta, S, T, \bar{B})$ be given. Any change in the endowment and social security benefit vector $(\omega, S)$ to some new vector $\left(\omega^{\prime}, S^{\prime}\right)$ which leaves the aggregate endowment and benefit levels $N \omega$ and $N S$ unchanged, and for which the resulting vector of parameters $e^{\prime}=\left(N, \omega^{\prime}, \alpha, \beta\right.$, $\left.S^{\prime}, T, \bar{B}\right)$ is an economy, will be called a distributional variation in $\omega$ and $S$. If $\left\|\left(\omega^{\prime}, S^{\prime}\right)-(\omega, S)\right\|<\varepsilon$ for some $\varepsilon>0$, where $\|\cdot\|$ denotes Euclidean distance in $R^{6}$, then the change will be referred to as an $\varepsilon$-distributional variation in $\omega$ and $S$. A subset of $R^{6}$ will be called a variational neighborhood of $\omega$ and $S$ if it contains all $\delta$-distributional variations in $\omega$ and $S$ for some $\delta>0$.

If $\left(p^{B^{\prime}}, i, M, B, c\right)$ is an equilibrium for $e$, and $(\omega, S) \rightarrow\left(\omega^{\prime}, S^{\prime}\right)$ is a distributional variation in $\omega$ and $S$, then $p^{B}$, $i$, and $\left(N c^{1}, N c^{2}, N c^{3}\right)$ will be said to be independent of this distributional variation if there exists an equilibrium $\left(p^{B}, i^{\prime}, M^{\prime}, B^{\prime}, c^{\prime}\right)$ for $e=\left(N, \omega^{\prime}, \alpha, \beta, S^{\prime}, T, \ddot{B}\right)$ satisfying $p^{B^{\prime}}=p^{B}, i^{\prime}$ $=i$, and $\left(N c^{1^{\prime}}, N c^{2^{\prime}}, N c^{3^{\prime}}\right)=\left(N c^{1}, N c^{2}, N c^{3}\right)$.

Analogous definitions will be used below for distributional variations in $S$ alone and in $\omega$ alone, and for independence with respect to such variations.

The following theorem investigates the impact of distributional variations on the real price of bonds $p^{B}$, the bond interest rate $i$, and the aggregate consumption and money holding profiles $\left(N c^{1}, N c^{2}, N c^{3}\right)$ and $\left(N M^{1}, N M^{2}\right)$ for each generation. Note that the invariance of the aggregate consumption profile to distributional variations implies the invariance of aggregate consumption at each time $t$ to distributional variations, and similarly for the aggregate money-holding profile.

Distribution neutrality theorem 4.2. Let an economy $e=(N, \omega, \alpha, \beta, S, T, \bar{B})$ be given with $N S>0$, and let $\left(p^{B}, i, M, B, C\right)$ be an equilibrium for $e$.

1. If $i=0$ over some variational neighborhood of $\omega$ and $S$, then $p^{B}, i$, and $\left(N c^{1}\right.$, $N c^{2}, N c^{3}$ ) are independent of all $c$-distributional variations in $\omega$ and $S$ for some $\varepsilon>0$, and $\left(N M^{1}, N M^{2}\right)$ is independent of all $\varepsilon^{\prime}$-distributional variations in $S$ for some $\varepsilon^{\prime}>0$.

2. If $i>0$, then $p^{B}, i$, and $\left(N c^{1}, N c^{2}, N c^{3}\right)$ are independent of all \&-distributional variations in $\omega$ and $S$ for some $\varepsilon>0$ if and only if all agents initially hold positive money balances only in their youth, i.e. if and only if $\left(M_{k}^{1}>0, M_{k}^{2}\right.$ $=0, k=1,2$ ).

3. If $i>0$, then $p^{B}, i,\left(N c^{1}, N c^{2}, N c^{3}\right)$, and $\left(N M^{1}, N M^{2}\right)$ are independent of all $\varepsilon$-distributional variations in $S$ for some $\varepsilon>0$ if and only if all agents initially exhibit qualitatively identical liquidity preference behavior in the sense that 
either $\left(M_{k}^{1}>0, M_{k}^{2}=0, k=1,2\right)$, or $\left(M_{k}^{1}=M_{k}^{2}=0, k=1,2\right)$, or $\left(M_{k}^{1}=0\right.$, $\left.M_{k}^{2}>0, k=1,2\right)$ over some variational neighborhood of $S$.

Remark. The various conditions of theorem 4.2 can be readily translated into restrictions on primitive parameters via theorems 3.2 through 3.5 in appendix $\mathrm{A}$.

Various existence, uniqueness, and distributional neutrality results will now be derived for the special cases of zero youth endowments $\left(\omega_{1}^{1}, \omega_{2}^{1}\right)$, zero middle-age endowments $\left(\omega_{1}^{2}, \omega_{2}^{2}\right)$, and zero net aggregate bonds $\bar{B}$, respectively. The first result, theorem 4.3 , indicates the very special distributional neutrality properties of economies with positive youth endowments only. Specifically, all macro variables are invariant with respect to all distributional variations in benefits and youth endowments, both local and global. Theorem 4.3 is illustrated in fig. $3 .^{4}$

Theorem 4.3. (Existence and distribution neutrality with youth endowments only). Let an economy $e=(N, \omega, \alpha, \beta, S, T, \bar{B})$ be given for which $N \omega^{1}>0$, $N \omega^{2}=0$, and $T N S>0$.

1. If $(\bar{B}+N S) T<\beta N S /(1+\alpha+\beta)$, there exist infinitely many equilibria for $e$; and if $(\bar{B}+N S) T=\beta N S /(1+\alpha+\beta)$, there exists a unique equilibrium for $e$. For all such equilibria, $i=0$ and $M_{k}^{1}>M_{k}^{2}, k=1,2$.

2. If $(\bar{B}+N S) T>\beta N S /(1+\alpha+\beta)$ there exists a unique equilibrium for $e$, with $i>0$ and $\left(M_{k}^{1}>0, M_{k}^{2}=0\right), k=1,2$.

3. Given any equilibrium $\left(p^{B}, i, M, B, c\right)$ for $e$, the real price of bonds $p^{B}$, bond interest rate $i$, and aggregate consumption and money holding profiles $\left(\mathrm{Nc}^{1}\right.$, $\left.N c^{2}, N c^{3}\right)$ and $\left(N M^{1}, N M^{2}\right)$ are independent of all distributional variations in $S$ and $\left(\omega_{1}^{1}, \omega_{2}^{1}\right)$, both local and global.

The analysis of economies with positive youth endowments and zero middle-age endowments is much simpler than the analysis of economics with zero youth endowments and positive middle-age endowments. The technical difficulty in the latter case is that the interest rate $i$ in theorem 3.8 cannot be represented in a convenient analytical form, resulting in a region described

\footnotetext{
${ }^{4}$ The precise derivation of fig. 3 and the ensuing figs. 4-6 is as follows. First, consider the stratification induced on the space $\xi$ of all economies $e$ by the stratification of competitive equilibria in accordance with distinct liquidity preference behavior, as characterized by theorems 3.1-3.8. Next, consider that stratified subset of $\xi$ compatible with the additional specified parameter restrictions (e.g. $N \omega^{1}>0, N \omega^{2}=0$, and $\bar{B}<0$ for fig. 3). Finally, project this stratified subset of $\xi$ onto the specified two-dimensional subspace (e.g. $N S-T$ space for fig. 3 ). Each region number in the resulting two-dimensional stratification (e.g. 3.2 and 3.3 in fig. 3) refers to the particular section 3 existence theorem giving the analytical characterization of the region in terms of primitive parameter restrictions. Note that $i>0$ in each region unless otherwise indicated.
} 


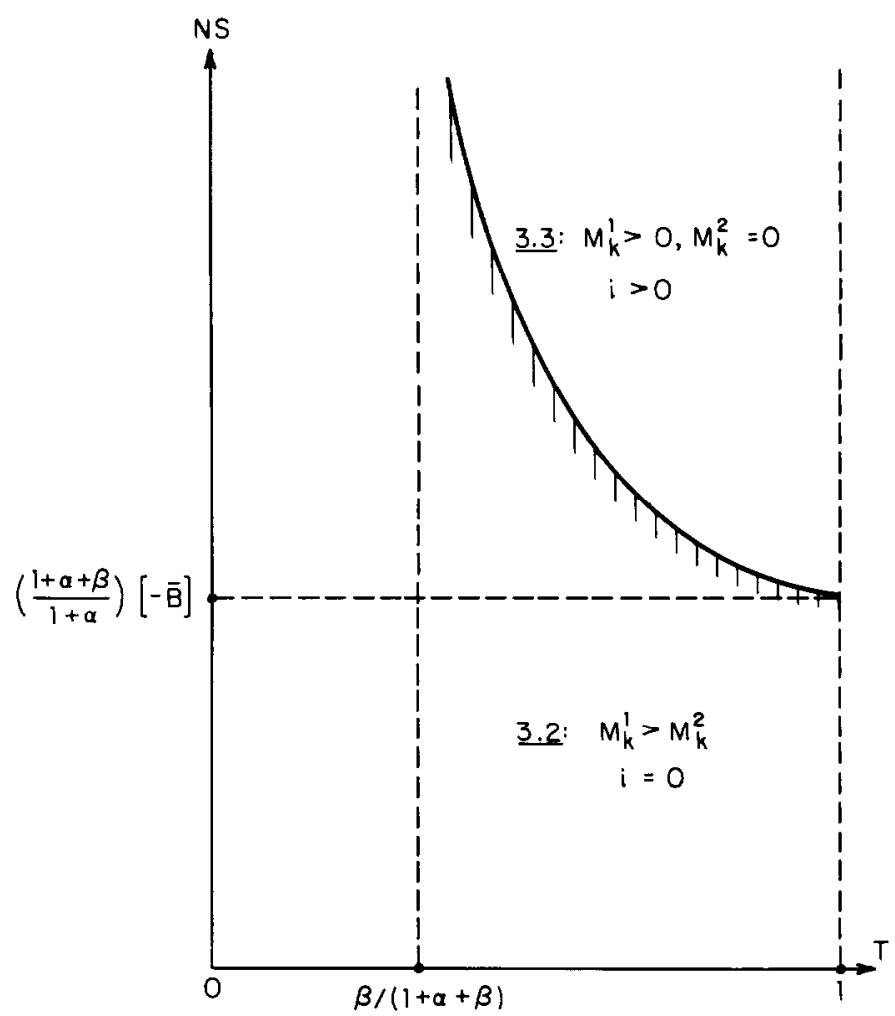

Fig. 3. Illustration of theorem 4.3 (existence of equilibria with $N \omega^{1}>0$ and $N \omega^{2}=0$ ) for arbitrary fixed discount and bond values $\alpha, \beta$, and $\bar{B}<0$, and for variable tax rate $T$ and aggregate social security benefits NS.

by quartic inequalities. The relatively less tractable nature of the equilibria described by theorem 3.8 is perhaps not surprising, since the hypotheses of theorem 3.8 define the set of economies for which agent types 1 and 2 exhibit the least similar liquidity preference behavior, i.e. $\left(M_{1}^{1}=0, M_{1}^{2}>0\right)$ versus $\left(M_{2}^{1}>0, M_{2}^{2}=0\right)$.

As the following theorem demonstrates, it is nevertheless still possible to determine a general necessary condition for existence for the case of zero youth endowments and positive middle-age endowments. Specifically, government must buy bonds from young agents in each period $t$ (i.e. $\bar{B} \leqq 0$ ), and the amount of such bonds must exceed the aggregate level $N S$ of social security benefits distributed to old agents in each period $t$ by a factor of $(1+\beta)$. Thus, unlike the case of positive youth endowments and zero middleage endowments treated in theorem 4.3, government is unable to set aggregate social security benefits at arbitrarily high levels without destroying stationary equilibrium. 
Theorem 4.4. (Existence region with middle-age endowments only). Let an economy $e=(N, \omega, \alpha, \beta, S, T, \bar{B})$ be given with $N \omega^{1}=0$ and $N \omega^{2}>0$. Then a necessary condition for the existence of an equilibrium for $e$ is

$$
N S \leqq(1+\beta)[-\bar{B}] .
$$

Remark. Condition (6) is not sufficient for the existence of an equilibrium for $e$, as can be seen by considering the special case $N_{1}=N_{2}=1, \omega_{1}^{1}=\omega_{2}^{1}=0$, $\omega_{1}^{2}=\omega_{2}^{2}>0, \alpha=\beta=1, S_{1}=0, S_{2}=1.75, T=1 / 2$, and $\bar{B}=-1$.

An example is depicted in fig. 4. All regions apart from $A$ and $A^{*}$ can be determined analytically. It can also be analytically established that each

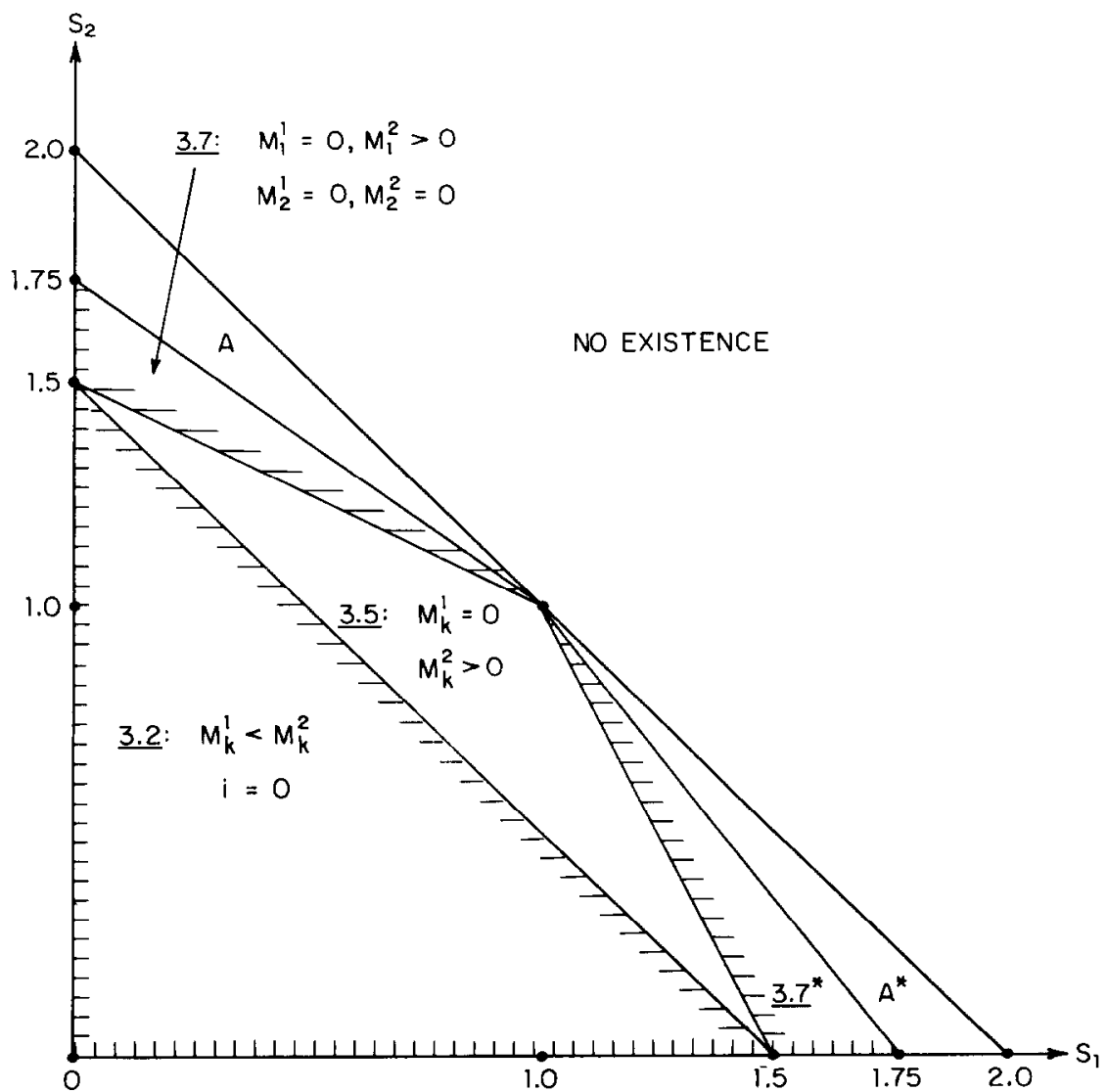

Fig. 4. Existence of equilibria with $N \omega^{1}-0, N \omega^{2}>0$, and $T N S>0$ for the special case $N_{1}=N_{2}$ $=1, \omega_{1}^{2}=\omega_{2}^{2}, \alpha=\beta=1, T=1 / 2$, and $\bar{B}=-1$. 
point in $A$ or $A^{*}$ is either a point of nonexistence, or a point satisfying the conditions of theorem 3.8 or its symmetrical counterpart $3.8^{*}$ respectively. (A numerical search of region $A$ revealed no points satisfying the hypotheses of theorem 3.8.) The general geometric configuration of the liquidity preference regions depicted in fig. 4 is intuitively reasonable: given $N \omega^{1}=0$, young agents must sell two-period bonds to finance their consumption. They will therefore plan to carry over money from middle to old age to help repay their debts, unless their old-age security benefits are sufficiently large.

As the following theorem 4.5 demonstrates, active government intervention in the economy is necessary for the existence of equilibria only in the case of zero youth endowments. Theorem 4.5 is illustrated in fig. 5 .

Theorem 4.5. (Existence and distribution neutrality with no government intervention). Let an economy $e=(N, \omega, \alpha, \beta, S, T, \bar{B})$ be given with $N S=T$ $=\bar{B}=0$. Then infinitely many equilibria exist for e if $N \omega^{1}>0$, and no equilibria exist for $e$ if $N \omega^{1}=0$. In any equilibrium for $e$, the interest rate $i$ is independent of all $\varepsilon$-distributional variations in $\omega$ for some $\varepsilon>0$ only if $i=0$.

Suppose government now considers the provision of social security benefits to type 1 agents only, i.e. $S_{1} \geqq 0$ and $S_{2}=0$. How does the set of equilibria vary as a function of $S_{1}$ ?

To examine this question, consider a zero net aggregate bond economy $e$ $=(N, \omega, \alpha, \beta, S, T, 0)$ for which complete symmetry prevails apart from the levels set for $S_{1}$ and $S_{2}$, i.e. $N_{1}=N_{2}, \omega_{1}^{1}=\omega_{2}^{1} \equiv \bar{\omega}$, and $\omega_{1}^{2}=\omega_{2}^{2} \equiv \overline{\bar{\omega}}$. By theorem 4.1 (endowment neutrality), the normalization $\overline{\bar{\omega}}=1-\bar{\omega}, 0 \leqq \bar{\omega} \leqq 1$, can be imposed on $e$ without loss of generality. By theorem 4.1 (money neutrality), an equilibrium exists for $e$ if and only if it exists for $e_{\lambda}=(N, \omega, \alpha$, $\beta, \lambda S, T, 0)$ for all $\lambda>0$, and the corresponding equilibrium real present valuc $p^{B} \lambda N S$ of social security benefits is independent of $\lambda$.

The following theorem 4.6 establishes a stronger existence and $S_{1}$ neutrality result for $e$. Briefly, an equilibrium exists for $e$ if and only if $S_{1}>0$, and the number of equilibria corresponding to $e$ is independent of $S_{1}$ over $R_{++}$.

Theorem 4.6. (Existence and $S_{1}$-distribution neutrality with government intervention only on behalf of type 1 agents). Let a zero aggregate net bond economy $e=(N, \omega, \alpha, \beta, S, T, 0)$ be given with $N_{1}=N_{2}, \omega_{1}^{1}=\omega_{2}^{1} \equiv \bar{\omega}$, and $\omega_{1}^{2}$ $=\omega_{2}^{2}=1-\bar{\omega}$.

1. Suppose $S_{1}=S_{2}=0$. Then, given $T=0$, infinitely many zero interest rate equilibria exist for $e$ if $1 /(1+\alpha+\beta) \leqq \bar{\omega} \leqq 1$, and a unique positive interest rate equilibrium exists for $e$ if $0<\bar{\omega}<1 /(1+\alpha+\beta)$. No equilibria exist for $e$ if either $T>0$ or $\bar{\omega}=0$. 


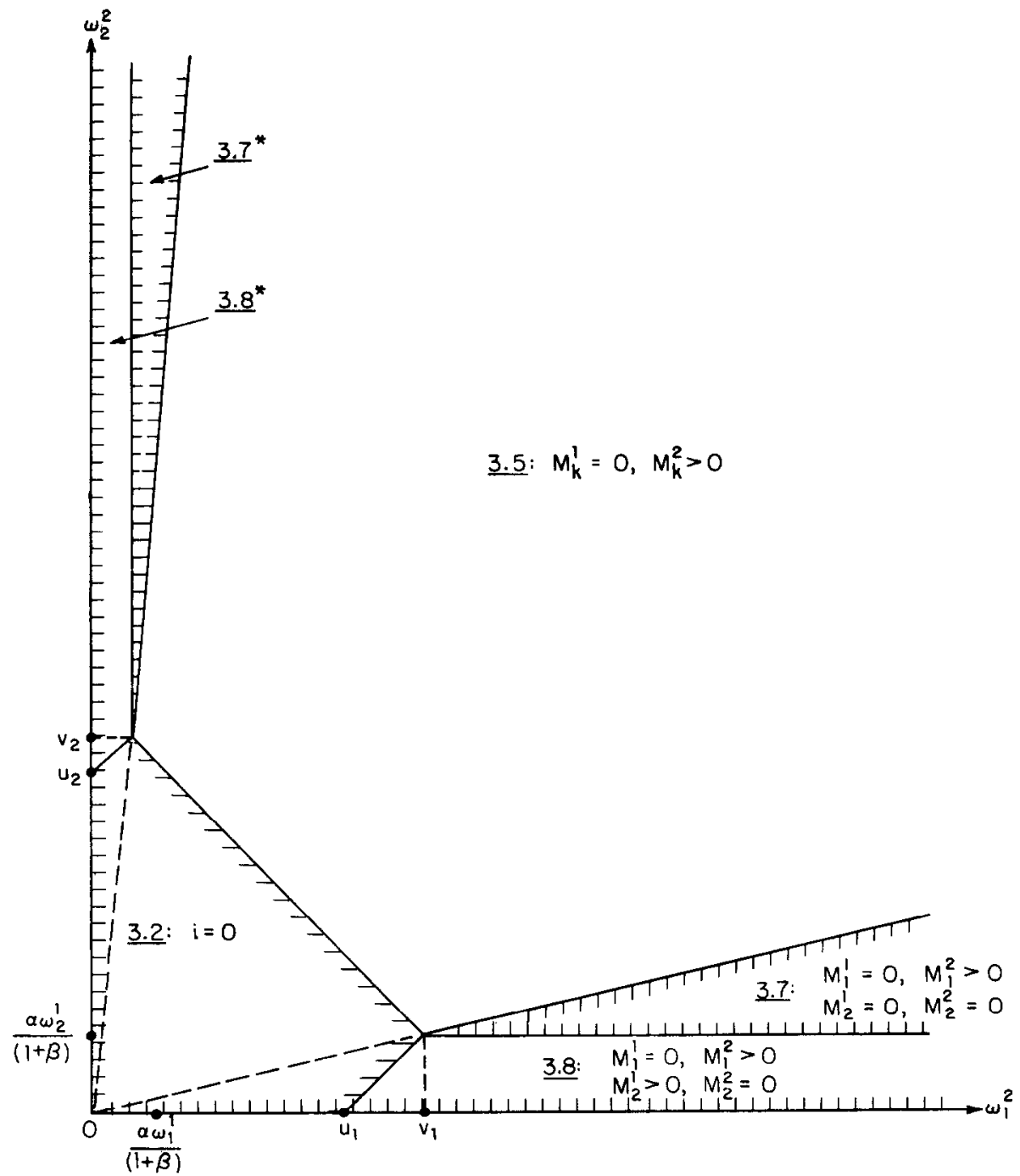

Fig. 5. Illustration of theorem 4.6 (existence of equilibria with $N S=T=\bar{B}=0$ ), where $u_{1} \equiv(\alpha$ $+\beta) \omega_{1}^{1}+\left(\beta N_{2} / N_{1}\right) \omega_{2}^{1}, \quad v_{1} \equiv u_{1}+\left(\alpha \beta N_{2} /(1+\beta) N_{1}\right) \omega_{2}^{1}, \quad u_{2} \equiv(\alpha+\beta) \omega_{2}^{1}+\left(\beta N_{1} / N_{2}\right) \omega_{1}^{1}, \quad$ and $\quad v_{2} \equiv u_{2}$ $+\left(\alpha \beta N_{1} /(1+\beta) N_{2}\right) \omega_{1}^{1}$.

2. Suppose $S_{1}>0$ and $S_{2}=0$. Then, given $T>0$ and $\bar{\omega}>0$, infinitely many zero interest rate equilibria exist for $e$ if $e$ satisfies

$$
\begin{aligned}
& T \leqq 1 /(1+\alpha+\beta), \\
& \bar{\omega} \geqq \min \left\{\frac{1}{(1+\alpha+\beta)[1-T]}, \frac{(1+2 \alpha+\beta) T+(1-\beta)}{(1+\alpha+\beta)[1-T]}\right\},
\end{aligned}
$$


with strict inequalities, a unique zero interest rate equilibrium exists for $e$ if $e$ satisfies conditions (7) with at least one equality, and a unique positive interest rate equilibrium exists for e othernise. No equilibria exist for e if $T$ $=0$ or $\bar{\omega}-0$.

Part 2 of theorem 4.6 is depicted in fig. 6. The points $F, G$, and $I$ are given by

$$
\begin{aligned}
& F \equiv\left(\frac{\beta}{1+2 \alpha+\beta}, \frac{1+2 \alpha+\beta}{(1+2 \alpha)(1+\alpha+\beta)}\right), \\
& G \equiv\left(\frac{\beta}{1+\alpha+\beta}, \frac{1+\beta}{1+\alpha+\beta}\right), \\
& I \equiv\left(\frac{\beta(1+\beta)(1+2 \beta)}{[\alpha+\beta(1+\beta)][1+2 \beta]+[1+\beta]}, \frac{1+\beta}{1+\alpha+\beta+2 \alpha \beta}\right) .
\end{aligned}
$$

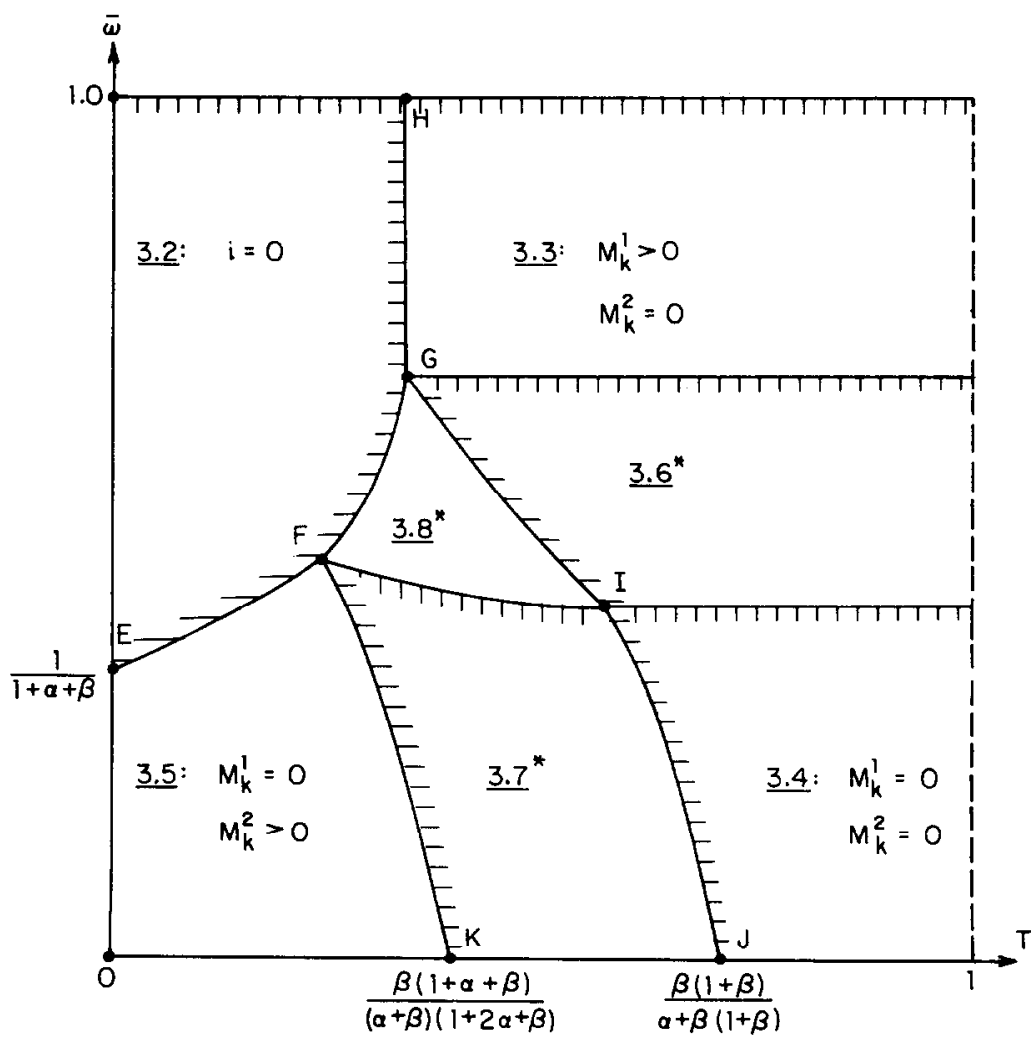

Fig. 6. Existence of equilibria with $S_{1}>0$ and $S_{2}=\bar{B}=0$ for the case $N_{1}=N_{2}, \omega_{1}^{1}=\omega_{2}^{1} \equiv \omega$, and $\omega_{1}^{2}=\omega_{2}^{2}=1-\bar{\omega}$. 
Note that all indicated partition points in fig. 6 are independent of $S_{1}$, illustrating the surprising fact that agent liquidity preference behavior is qualitatively invariant with respect to the magnitude of the nominal social security benefits $S_{1}$ allocated to the favored type 1 agent as long as these benefits are positive.

\section{Appendix A: Section 3. Existence and uniqueness theorems}

Theorem 3.1. (Impossibility of two-period positive money holding with positive interest rate). Given any economy $e=(N, \omega, \alpha, \beta, T, \bar{B})$, there exists no equilibrium $\left(p^{B}, i, M, B, c\right)$ for $e$ such that $i>0$ and either $\left(M_{1}^{1}>0, M_{1}^{2}>0\right)$ or $\left(M_{2}^{1}>0, M_{2}^{2}>0\right)$.

Theorem 3.2. (Lero interest rate equilibria). Let an economy $e=(N, \omega, \alpha, \beta, S$, $T, \bar{B})$ be given.

1. If $0<T N S$, there exists a zero interest rate equilibrium $\left(p^{B}, 0, M, B, c\right)$ for $e$ if and only if

$$
\begin{array}{ll}
{\left[\frac{(1+\alpha+\beta) \bar{B}_{k}+S_{k}}{N S}\right] \leqq\left[\frac{1-T}{T}\right]\left[\frac{(\alpha+\beta) \omega_{k}^{1}-\omega_{k}^{2}}{N \omega}\right],} & k=1,2, \\
{\left[\frac{(1+\alpha+\beta) \bar{B}_{k}+(1+\alpha) S_{k}}{N S}\right] \leqq \beta\left[\frac{1-T}{T}\right]\left[\frac{\omega_{k}^{1}+\omega_{k}^{2}}{N \omega}\right], \quad k=1,2,}
\end{array}
$$

for some values $\bar{B}_{1}$ and $\bar{B}_{2}$ in $R$ satisfying $N_{1} \bar{B}_{1}+N_{2} \bar{B}_{2}=\bar{B}$. For each such pair, the equilibrium is unique.

2. If $0=T=N S$, then any value $p>0$ satisfying

$$
p(1+\alpha+\beta) \bar{B}_{k} \leqq \min \left\{(\alpha+\beta) \omega_{k}^{1}-\omega_{k}^{2}, \beta\left[\omega_{k}^{1}+\omega_{k}^{2}\right]\right\}, \quad k=1,2,
$$

for some $\bar{B}_{1}$ and $\bar{B}_{2}$ in $R$ with $N_{1} \bar{B}_{1}+N_{2} \bar{B}_{2}=\bar{B}$ yields an equilibrium price $p^{B}=p$ for $e$ with corresponding equilibrium interest rate $i=0$.

3. No zero interest rate equilibria exist for e otherwise.

Corollary 3.2.1. The subset of economies with infinitely many equilibria has positive Lebesgue measure in $R^{12}$.

Theorem 3.3. (Equilibria with youth money holding only). Let an economy $e$ $=(N, \omega, \alpha, \beta, S, T, \bar{B})$ be given. There exists an equilibrium $\left(p^{B}, i, M, B, c\right)$ for 
e satisfying $i>0$ and $\left(M_{k}^{1}>0, M_{k}^{2}=0\right), k=1,2$, if and only if

$$
\begin{aligned}
& \omega_{k}^{2}<\left(\frac{\alpha}{1+\beta}\right)\left[\left(\omega_{k}^{1}+p^{B}[1-T]^{-1} S_{k}\right], \quad k=1,2,\right. \\
& \left(\frac{\beta}{1+\alpha+\beta}\right) N S<(\bar{B}+N S) T,
\end{aligned}
$$

with

$p^{B} \equiv \frac{\beta[1-T] N \omega}{(1+\alpha+\beta) \bar{B}+(1+\alpha) N S}, \quad i-\left(\frac{1+\alpha+\beta}{\beta[1-T]}\right)\left[T-\left(\frac{\beta}{1+\alpha+\beta}\right)\left[\frac{N S}{\bar{B}+N S}\right]\right]$.

Given existence, the equilibrium is unique.

Theorem 3.4. (Equilibria with zero money holding). Let an economy $e=(N, \omega)$, $\alpha, \beta, S, T, \bar{B})$ be given. Define $D \equiv(1+\beta) \bar{B}+N S$.

1. If $D \neq 0$, there exists an equilibrium $\left(p^{B}, i, M, B, c\right)$ for $e$ satisfying $i>0$ and $\left(M_{k}^{1}=0, M_{k}^{2}=0\right), k=1,2$, if and only if

$$
\begin{aligned}
& \left(\frac{\alpha}{1+\beta}\right)\left[\omega_{k}^{1}+p^{B}[1-T]^{-1} S_{k}\right] \leqq \omega_{k}^{2} \leqq(1+i)\left(\frac{\alpha}{1+\beta}\right)\left[\omega_{k}^{1}+p^{B}[1-T]^{-1} S_{k}\right], \\
& 0<D N \omega^{1}, \\
& \frac{\beta N S N \omega^{1}}{\beta N S N \omega^{1}+D N \omega}<T,
\end{aligned}
$$

where

$$
p^{B} \equiv \frac{1}{D}\left(\beta[1-T] N \omega^{1}\right), \quad i \equiv\left(\frac{1+\beta}{\beta}\right)\left[\frac{T N \omega-p^{B} N S}{\lfloor 1-T\rfloor N \omega^{1}+p^{B} N S}\right]
$$

Given existence, the equilibrium is unique.

2. If $D=0, N \omega^{1}=0$, and $0<\alpha S_{k} T N \omega^{2}-\beta N S[1-T] \omega_{k}^{2}, k=1,2$, then any value $p>0$ satisfying

$$
p \leqq \min \left\{\frac{(1+\beta)[1-T] \omega_{k}^{2}}{\alpha S_{k}}, \frac{(1+\beta)\left[\alpha S_{k} T N \omega^{2}-\beta N S[1-T] \omega_{k}^{2}\right]}{\alpha S_{k} N S}\right\}
$$

$$
k=1,2 \text {, }
$$


yields an equilibrium price $p^{B}=p$ for $e$ with $i>0$ as in part 1 , and with $M_{k}^{1}$ $=M_{k}^{2}=0, k=1,2$.

3. No zero money holding equilibria with $i>0$ exist for e otherwise.

Theorem 3.5. (Equilibria with middle-age money holding only). Let an economy $e=(N, \omega, \alpha, \beta, S, T, \bar{B})$ be given, and define $Q \equiv(1+\alpha+\beta) B+N S$.

1. If $Q \neq 0$, there exists an equilibrium $\left(p^{B}, i, M, \beta, c\right)$ for $e$ satisfying $i>0$ and $\left(M_{k}^{1}=0, M_{k}^{2}>0\right), k=1,2$, if and only if

$$
\begin{aligned}
& (1+i)\left(\frac{\alpha}{1+\beta}\right)\left[\omega_{k}^{1}+p^{B}[1-T]^{-1} S_{k}\right]<\omega_{k}^{2}, \quad k=1,2, \\
& 0<p^{B}<\infty, \quad 0<i<\infty
\end{aligned}
$$

where

$$
\begin{aligned}
& p^{B} \equiv \frac{[1-T]}{(1+i) Q}\left[(1+i)(\alpha+\beta) N \omega^{1}-N \omega^{2}\right], \\
& (1+i) \equiv \begin{cases}\left(Y \pm \sqrt{Y^{2}-4 X Z}\right) / 2 X, & \text { if } X \neq 0, \\
Z / Y, & \text { if } X=0 \text { and } Y \neq 0, \\
+\infty, & \text { otherwise, }\end{cases} \\
& X \equiv(\alpha+\beta)(\bar{B}+N S)[1-T] N \omega^{1}, \\
& Y \equiv[(\alpha+\beta) \bar{B}[1-T]+Q T] N \omega^{1}+[(\bar{B}+N S)[1-T]+Q T] N \omega^{2}, \\
& Z \equiv \bar{B}[1-T] N \omega^{2} .
\end{aligned}
$$

2. If $Q=0$ and $T N S>0$, there exists an equilibrium for e satisfying $i>0$ and $\left(M_{k}^{1}=0, M_{k}^{2}>0\right), k=1,2$, if and only if $0<(\alpha+\beta) N \omega^{1}<N \omega^{2}$ and condition (A3) in part 1 holds for $p^{B}$ and $i$, where

$$
p^{B} \equiv \frac{(1+\alpha+\beta) T N \omega^{1}}{N S}, \quad i \equiv \frac{N \omega^{2}-(\alpha+\beta) N \omega^{1}}{(\alpha+\beta) N \omega^{1}}
$$

Given existence, the equilibrium is unique.

3. If $N S=T=\bar{B}=0, \quad 0<(\alpha+\beta) N \omega^{1}<N \omega^{2}, \quad$ and $\left[\alpha N \omega^{2}\right] \omega_{k}^{1}<(1+\beta)(\alpha+\beta)$ 
$\times N \omega^{1} \omega_{k}^{2}, k=1,2$, then any value $p>0$ yields an equilibrium price $p^{B}=p$ for e with

$$
i=\frac{N \omega^{2}-(\alpha+\beta) N \omega^{1}}{(\alpha+\beta) N \omega^{1}}>0
$$

and $\left(M_{k}^{1}=0, M_{k}^{2}>0\right), k=1,2$.

4. No equilibria with $i>0$ and $\left(M_{k}^{1}=0, M_{k}^{2}>0\right), k=1,2$, exist for e otherwise.

Remark. Since

$$
Y^{2}-4 X Z=V^{2}+4 Q^{2} T N \omega^{1} N \omega^{2} \geqq 0,
$$

where

$$
V \equiv[(\alpha+\beta) \bar{B}+(\bar{B}+N S) T] N \omega^{1}-[(\alpha+\beta) \bar{B} T+(\bar{B}+N S)] N \omega^{2}
$$

the quadratic roots $i$ defined by (A4) are real. It follows by Descartes' rule of signs (for polynomials with real roots) that the number of positive solutions $i$ for (A4) is precisely equal to the number of successive sign changes in the nonzero coefficients of

$$
0=i^{2} X+i[2 X-Y]+[X+Z-Y] \text {. }
$$

Since

$$
Y=X+Z+Q T N \omega+N S[1-T]\left(N \omega^{2}-(\alpha+\beta) N \omega^{1}\right),
$$

a sufficient condition for the existence of a unique positive value $i$ satisfying (A4) is thus, for example, $N S=0, T N \omega^{1}>0$, and $\bar{B} \neq 0$; for then $X$ and $Q T N \omega$ have the same sign, implying that $X$ and $X+Z-Y$ have opposite signs. The nonvacuity of theorem 3.5 (part 1) can be seen by considering, for example, $N_{1}=N_{2}=1 / 2, \omega_{1}^{1}=\omega_{2}^{1}=0, \omega_{1}^{2}=\omega_{2}^{2}>0, \alpha=\beta=1$, $S_{1}=S_{2}=1,0<T<1 / 3$, and $\bar{B}=-1$.

Theorem 3.6. (Equilibria with weakly dissimilar youth money holding). Let an economy $e=(N, \omega, \alpha, \beta, S, T, \bar{B})$ be given. There exists an equilibrium $\left(p^{B}, i, M\right.$, $B, c)$ for e satisfying $i>0,\left(M_{1}^{1}=0, M_{1}^{2}=0\right)$, and $\left(M_{2}^{1}>0, M_{2}^{2}=0\right)$ if and only if

$$
\left(\frac{\alpha}{1+\beta}\right)\left[\omega_{1}^{1}+p^{B}[1-T]^{-1} S_{1}\right] \leqq \omega_{1}^{2} \leqq(1+i)\left(\frac{\alpha}{1+\beta}\right)\left[\omega_{1}^{1}+p^{B}[1-T]^{-1} S_{1}\right],
$$




$$
\begin{aligned}
& \omega_{2}^{2}<\left(\frac{\alpha}{1+\beta}\right)\left[\omega_{2}^{1}+p^{B}[1-T]^{-1} S_{2}\right], \\
& 0<\left(\frac{1}{1+\beta}\right) N_{1} S_{1}+\left(\frac{1+\alpha}{1+\alpha+\beta}\right) N_{2} S_{2}+\bar{B}, \\
& 0<T N \omega-p^{B} N S,
\end{aligned}
$$

where

$$
\begin{aligned}
& p^{B} \equiv \frac{\beta[1-T]\left[(1+\alpha+\beta) N_{1} \omega_{1}^{1}+(1+\beta) N_{2} \omega_{2}\right]}{(1+\beta)(1+\alpha+\beta) \bar{B}+(1+\alpha+\beta) N_{1} S_{1}+(1+\alpha)(1+\beta) N_{2} S_{2}}, \\
& i \equiv \frac{T N \omega-p^{B} N S}{p^{B}[\bar{B}+N S]} .
\end{aligned}
$$

Given existence, the equilibrium is unique.

Remark. Theorem 3.6 with the roles of agent types 1 and 2 interchanged will be referred to as theorem $3.6^{*}$.

Theorem 3.7. (Equilibria with weakly dissimilar middle-age money holding). Let an economy $e=(N, \omega, \alpha, \beta, S, T, \bar{B})$ be given, and define $W \equiv(1$ $+\alpha+\beta)[(1+\beta) \bar{B}+N S]-\alpha N_{1} S_{1}$ and $L \equiv(1+\beta)(\alpha+\beta) N_{1} \omega_{1}^{1}+\beta(1+\alpha+\beta) N_{2} \omega_{2}^{1}$.

1. If $W \neq 0$, there exists an equilibrium $\left(p^{B}, i, M, B, c\right)$ for $e$ satisfying $i>0$, $\left(M_{1}^{1}=0, M_{1}^{2}>0\right)$, and $\left(M_{2}^{1}=0, M_{2}^{2}=0\right)$ if and only if

$$
\begin{aligned}
& (1+i)\left(\frac{\alpha}{1+\beta}\right)\left[\omega_{1}^{1}+p^{B}[1-T]^{-1} S_{1}\right]<\omega_{1}^{2}, \\
& \left(\frac{\alpha}{1+\beta}\right)\left[\omega_{2}^{1}+p^{B}[1-T]^{-1} S_{2}\right] \leqq \omega_{2}^{2} \leqq(1+i)\left(\frac{\alpha}{1+\beta}\right)\left[\omega_{2}^{1}+p^{B}[1-T]^{-1} S_{2}\right] \\
& 0<p^{B}<\infty, \quad 0<i<\infty,
\end{aligned}
$$

where

$$
p^{B} \equiv \frac{[1-T]}{(1+i) W}\left((1+i) L-(1+\beta) N_{1} \omega_{1}^{2}\right)
$$




$$
\begin{aligned}
&(1+i) \equiv \begin{cases}\left(Y \pm \sqrt{Y^{2}-4 X Z}\right) / 2 X, & \text { if } X \neq 0, \\
Z / Y, & \text { if } X=0 \\
+\infty, & \text { otherwise, } Y \neq 0,\end{cases} \\
& X \equiv[\bar{B}+N S][1-T] L, \\
& Y \equiv[(1+\beta)(\alpha+\beta) \bar{B}[1-T]+W T] N_{1} \omega_{1}^{1} \\
&+ {[(1+\beta)(\bar{B}+N S)[1-T]+W T] N_{1} \omega_{1}^{2} } \\
&+[\beta(1+\alpha+\beta) \bar{B}[1-T]+W T] N_{2} \omega_{2}^{1} \\
&+[W T] N_{2} \omega_{2}^{2}, \\
& Z \equiv(1+\beta) \bar{B}[1-T] N_{1} \omega_{1}^{2} .
\end{aligned}
$$

2. If $W=0$ and $T N S>0$, there exists an equilibrium for e satisfying $i>0,\left(M_{1}^{1}\right.$ $\left.=0, M_{1}^{2}>0\right)$, and $\left(M_{2}^{1}=0, M_{2}^{2}=0\right)$ if and only if $0<L<(1+\beta) N_{1} \omega_{1}^{2}$ and conditions $(A 5)$ and $(A 6)$ hold for $p^{B}$ and $i$, where

$$
p^{B} \equiv \frac{L T N \omega}{(1+\beta)(\bar{B}+N S) N_{1} \omega_{1}^{2}+[-\bar{B}] L}, \quad i \equiv \frac{(1+\beta) N_{1} \omega_{1}^{2}-L}{L} .
$$

Given existence, the equilibrium is unique.

3. If $N S=T=\bar{B}=0, \quad 0<L<(1+\beta) N_{1} \omega_{1}^{2}, \quad \alpha N_{2} \omega_{2}^{1} \leqq(1+\beta) N_{2} \omega_{2}^{2}, \quad$ and $L N_{2} \omega_{2}^{2} \leqq\left[\alpha N_{1} \omega_{1}^{2}\right] N_{2} \omega_{2}^{1}$, then any value $p>0$ yields an equilibrium price $p^{B}$ $=p$ for $e$ with $i=\left[(1+\beta) N_{1} \omega_{1}^{2}-L\right] / L>0,\left(M_{1}^{1}=0, M_{1}^{2}>0\right)$, and $\left(M_{2}^{1}=0\right.$, $M_{2}^{2}=0$ ).

4. No equilibria satisfying $i>0,\left(M_{1}^{1}=0, M_{1}^{2}>0\right)$, and $\left(M_{2}^{1}=0, M_{2}^{2}=0\right)$ exist for e otherwise.

Remark. Note that $Y=X+Z+W T N \omega+N S[1-T]\left[(1+\beta) N_{1} \omega_{1}^{2}-L\right]$. As in the remark following the statement of theorem 3.5, a sufficient condition for the existence of a unique positive value $i$ satisfying (A7) is $Y^{2} \geqq 4 X Z, N S=0$, $T N \omega^{1}>0$, and $\bar{B} \neq 0$. The nonvacuity of theorem 3.7 is established in section 4. The statement of theorem 3.7 with the roles of agent types 1 and 2 interchanged will be referred to as theorem $3.7^{*}$.

Theorem 3.8. (Equilibria with strongly dissimilar money holding). Let an 
economy $e=(N, \omega, \alpha, \beta, S, T, \bar{B})$ be given, and define $R \equiv(1+\alpha+\beta) \bar{B}+N_{1} S_{1}$ $+(1+\alpha) N_{2} S_{2}$ and $K \equiv(\alpha+\beta) N_{1} \omega_{1}^{1}+\beta N_{2} \omega_{2}$.

1. If $R \neq 0$, there exists an equilibrium $\left(p^{B}, i, M, B, c\right)$ for $e$ satisfying $i>0,\left(M_{1}^{1}\right.$ $\left.=0, M_{1}^{2}>0\right)$, and $\left(M_{2}^{1}>0, M_{2}^{2}=0\right)$ if and only if

$$
\begin{aligned}
& (1+i)\left(\frac{\alpha}{1+\beta}\right)\left[\omega_{1}^{1}+p^{B}[1-T]^{-1} S_{1}\right]<\omega_{1}^{2}, \\
& \omega_{2}^{2}<\left(\frac{\alpha}{1+\beta}\right)\left[\omega_{2}^{1}+p^{B}[1-T]^{-1} S_{2}\right] \\
& 0<p^{B}<\infty, \quad 0<i<\infty
\end{aligned}
$$

where

$$
\begin{aligned}
& p^{B} \equiv \frac{[1-T]}{(1+i) R}\left((1+i) K-N_{1} \omega_{1}^{2}\right), \\
& (1+i) \equiv \begin{cases}\left(Y \pm \sqrt{Y^{2}-4 X Z}\right) / 2 X, & \text { if } X \neq 0, \\
+\infty, & \text { if } X=0 \text { and } Y \neq 0,\end{cases} \\
& X \equiv(\bar{B}+N S)[1-T] K, \\
& Y \equiv[(\alpha+\beta) \bar{B}[1-T]+R T] N_{1} \omega_{1}^{1} \\
& \quad+[(\bar{B}+N S)[1-T]+R T] N_{1} \omega_{1}^{2}+[\beta \bar{B}[1-T]+R T] N_{2} \omega_{2},
\end{aligned}
$$

2. If $R=0$ and $T N S>0$, there exists an equilibrium for e satisfying $i>0,\left(M_{1}^{1}\right.$ $\left.=0, \quad M_{1}^{2}>0\right)$, and $\left(M_{2}^{1}>0, M_{2}^{2}=0\right)$ if and only if $0<K<N_{1} \omega_{1}^{2}$ and conditions $(A 8)$ and $(A 9)$ hold for $p^{B}$ and $i$, where

$$
p^{B} \equiv \frac{K T N \omega}{(\bar{B}+N S) N_{1} \omega_{1}^{2}+[-\bar{B}] K}, \quad i \equiv \frac{N_{1} \omega_{1}^{2}-K}{K} .
$$

Given existence, the equilibrium is unique.

3. If $N S=T=\bar{B}=0,0<K<N_{1} \omega_{1}^{2}$, and $(1+\beta) \omega_{2}^{2}<\alpha \omega_{2}^{1}$, then any value $p>0$ 
yields an equilibrium price $p^{B}=p$ for $e$ with $i=\left(N_{1} \omega_{1}^{2}-K\right) / K>0,\left(M_{1}^{1}=0\right.$, $\left.M_{1}^{2}>0\right)$, and $\left(M_{2}^{1}>0, M_{2}^{2}=0\right)$.

4. No equilibria with i>0, $\left(M_{1}^{1}=0, \quad M_{1}^{2}>0\right), \quad$ and $\quad\left(M_{2}^{1}>0\right.$, $M_{2}^{2}=0$ ) exist for $e$ otherwise.

Remark. Sincc $Y=X+Z+R T N \omega+N S[1-T]\left[N_{1} \omega_{1}^{2}-K\right]$, it follows, as in the remarks following theorems 3.5 and 3.7 , that a sufficient condition for the existence of a unique positive value $i$ satisfying (A10) is $Y^{2} \geqq 4 X Z, N S=0$, $T K>0$, and $\bar{B} \neq 0$. The nonvacuity of theorem 3.8 is established in section 4 . The statement of theorem 3.8 with the roles of agent types 1 and 2 interchanged will be referred to as theorem $3.8^{*}$.

\section{Appendix B: Proof outlines}

Proof of theorems 3.1-3.8. For each $k=1,2$, the young agent planning problem (1) is a concave programming problem subject to linear constraints. The following Kuhn-Tucker conditions (KT) are thus both necessary and sufficient for a solution for any given $p^{B}>0$ and $i>0$ :

(KT) There exists a vector $\left(M_{k}, B_{k}, c_{k}\right)$ in $R^{2} \times R \times R_{++}^{3}$ and a vector $(\lambda, \theta)$ in $R_{+}^{2}$ satisfying

$$
\begin{gathered}
c_{k}^{1}=[1-T] \omega_{k}^{1}-(1+i) p^{B} M_{k}^{1}-p^{B} B_{k}, \\
c_{k}^{2}=[1-T] \omega_{k}^{2}+(1+i) p^{B}\left[M_{k}^{1}-M_{k}^{2}\right], \\
c_{k}^{3}=(1+i) p^{B}\left[B_{k}+S_{k}+M_{k}^{2}\right], \\
{\left[\frac{(1+i) p^{B}}{[1-T] \omega_{k}^{1}-(1+i) p^{B} M_{k}^{1}-p^{B} B_{k}}+\frac{\alpha(1+i) p^{B}}{[1-T] \omega_{k}^{2}+(1+i) p^{B}\left[M_{k}^{1}-M_{k}^{2}\right]}+\lambda\right]=0,} \\
{\left[\frac{-\alpha(1+i) p^{B}}{[1-T] \omega_{k}^{2}+(1+i) p^{B}\left[M_{k}^{1}-M_{k}^{2}\right]}+\frac{\beta(1+i) p^{B}}{(1+i) p^{B}\left[B_{k}+S_{k}+M_{k}^{2}\right]}+\theta\right]=0,} \\
{\left[\frac{-p^{B}}{[1-T] \omega_{k}^{1}-(1+i) p^{B} M_{k}^{1}-p^{B} B_{k}}+\frac{\beta(1+i) p^{B}}{(1+i) p^{B}\left[B_{k}+S_{k}+M_{k}^{2}\right]}\right]=0,}
\end{gathered}
$$




$$
\begin{aligned}
& \lambda M_{k}^{1}=0, \quad M_{k}^{1} \geqq 0, \\
& \theta M_{k}^{2}=0, \quad M_{k}^{2} \geqq 0 .
\end{aligned}
$$

It follows from (KT) that a vector $\left(M_{k}, B_{k}, c_{k}\right)$ in $R_{++}^{2} \times R \times R_{++}^{3}$ is a solution to the planning problem (1) if and only if $c_{k}$ satisfies (B1) through (B3) and $\left(M_{k}, B_{k}\right)$ is a solution for the system of equations

$$
\begin{aligned}
& \left(\begin{array}{lll}
\alpha p^{B} & -(1+i) p^{B} & (1+\alpha)(1+i) p^{B} \\
\alpha(1+i) p^{B} & (\beta+\alpha)(1+i) p^{B} & -\beta(1+i) p^{B} \\
(1+\beta) p^{B} & p^{B} & \beta(1+i) p^{B}
\end{array}\right)\left(\begin{array}{l}
B_{k} \\
M_{k}^{2} \\
M_{k}^{1}
\end{array}\right) \\
& \quad=\left(\begin{array}{l}
\alpha[1-T] \omega_{k}^{1}-[1-T] \omega_{k}^{2} \\
\beta[1-T] \omega_{k}^{2}-\alpha(1+i) p^{B} S_{k} \\
\beta[1-T] \omega_{k}^{1}-p^{B} S_{k}
\end{array}\right) .
\end{aligned}
$$

Since the determinant $\alpha \beta(1+i) p^{B}\left[i p^{B}\right]^{2}$ of the Jacobian for systcm (B9) is nonzero, it can be shown that the unique solution for system (B9) is

$$
\begin{aligned}
& \left.B_{k}=L-i p^{B}\right\rfloor^{-1}\left([1-T]\left(\omega_{k}^{1}+\omega_{k}^{2}\right)+(1+i) p^{B} S_{k}\right), \\
& M_{k}^{2}=\left[i p^{B}\right]^{-1}\left([1-T]\left(\omega_{k}^{1}+\omega_{k}^{2}\right)+p^{B} S_{k}\right), \\
& M_{k}^{1}=\left[i p^{B}\right]^{-1}\left([1-T]\left[\omega_{k}^{1}+\frac{\omega_{k}^{2}}{(1+i)}\right]+p^{B} S_{k}\right) .
\end{aligned}
$$

However, the only consumption vector $c_{k}$ satisfying eqs. (B1) through (B3) for the bond and money holding values $(\mathrm{B} 10)$ is the zero vector. This establishes theorem 3.1 .

The proofs of theorems 3.3 through 3.8 are analogous to the proof of theorem 3.1. One first uses (KT) to find necessary and sufficient conditions for the existence of solutions to the young agent planning problem (1) having the stated liquidity preference characteristics, together with the precise form of these solutions, for any given real bond price $p^{B}>0$ and bond interest rate $i>0$. One then obtains necessary and sufficient conditions for the existence of positive values $p^{R}$ and $i$ consistent with both the solution forms and the market clearing conditions (3) and (4). Theorem 3.2 is proved similarly, after a change of variables $E_{k} \equiv B_{k}+M_{k}^{1}$ and $F_{k} \equiv B_{k}+M_{k}^{2}$. Q.E.D.

Proof of corollary 3.2.1. Suppose conditions (A1) hold with strict inequality for some economy $e^{*} \in R_{++}^{10} \times(0,1) \times R-\{0\}$ and some point

$$
\left(\bar{B}_{1}^{*}, \bar{B}_{2}^{*}\right) \in \mathscr{B}^{*} \equiv\left\{\left(B_{1}, B_{2}\right) \in R^{2} \mid N_{1} B_{1}+N_{2} B_{2}=\bar{B}^{*}\right\},
$$


where $\bar{B}^{*}$ is the last component of $e^{*}$. Then $e^{*}$ has infinitely many equilibria, since conditions (A1) continue to hold in some $R^{2}$ neighborhood $V^{*}$ of $\left(\bar{B}_{1}^{*}, \bar{B}_{2}^{*}\right)$ intersected with $\mathscr{B}^{*}$, and each point in $V^{*} \cap \mathscr{B}^{*}$ corresponds to a distinct equilibrium for $e^{*}$. On the other hand, for sufficiently small $\delta^{*}>0$, each vector of the form

$$
e_{\delta}^{*} \equiv \delta e^{*}, \quad|\delta-1|<\delta^{*},
$$

is an economy for which conditions (A1) also hold with strict inequality, with $\left(\delta \bar{B}_{1}^{*}, \delta \bar{B}_{2}^{*}\right)$ in place of $\left(\bar{B}_{1}^{*}, \bar{B}_{2}^{*}\right)$; hence, each economy (B11) also has infinitely many equilibria. It follows that $\left\{e_{\delta}^{*}|| \delta-1 \mid<\delta^{*}\right\}$ is an open rectangle of economies in $R^{12}$ with Lebesgue measure $\left(2 \delta^{*}\right)^{12}>0$ such that each element $e_{\delta}^{*}$ has infinitely many equilibria.

It thus suffices to exhibit an economy $e^{*}$ and point $\left(\bar{B}_{1}^{*}, \bar{B}_{2}^{*}\right)$ satisfying the required conditions. Such a pair is given by $e^{*} \equiv\left(N^{*}, \omega^{*}, \alpha^{*}, \beta^{*}, S^{*}, T^{*}, \bar{B}^{*}\right)$ and $\left(\bar{B}_{1}^{*}, \bar{B}_{2}^{*}\right)=\left(-\frac{1}{2},-\frac{1}{2}\right)$, where $N^{*}=(1,1), \omega^{*}=(1,1,1,1), \alpha^{*}=1, \beta^{*}=1, S^{*}$ $=(1,1), T^{*}=1 / 4$, and $\bar{B}^{*}=-1$. Q.E.D.

Proof of theorem 4.1. Using theorems 3.2 through 3.8, together with a symmetry argument to handle cases not explicitly covered, it can be verified that the necessary and sufficient conditions defining the various liquidity preference regions are satisfied by $e$ and $\left(p^{B}, i, M, B, c\right)$ if and only if they are satisfied by $e_{\lambda}$ and $\left(\lambda^{-1} p^{B}, i, \lambda M, \lambda B, c\right)$ for $\lambda>0$, where $\left(\lambda^{-1} p^{B}, i, \lambda M, \lambda B, c\right)$ has the proper solution form. Thus, money neutrality holds, as claimed in part 1 . The endowment and population neutrality properties claimed in parts 2 and 3 are similarly established. Q.E.D.

Proof of theorem 4.2. The proof of part 1 follows from theorem 3.2. Specifically, one has $i=0, N c^{1}=N \omega /(1+\alpha+\beta), N c^{2}=\alpha N c^{1}, N c^{3}=\beta N c^{1}$, and $p^{B}=T N \omega / N S$ for all distributional variations in $\omega$ and $S$ for which the necessary conditions (A1) for $i=0$ remain satisfied; and

$$
\begin{aligned}
& N M^{1}=\left(\frac{N S}{1+\alpha+\beta}\right)\left[\left(\frac{1-T}{T}\right)\left(\frac{(\alpha+\beta) N \omega^{1}-N \omega^{2}}{N \omega}\right)-1\right]-\bar{B}, \\
& N M^{2}=\left(\frac{N S}{1+\alpha+\beta}\right)\left[\frac{\beta[1-T]}{T}-(1+\alpha)\right]-\bar{B},
\end{aligned}
$$

for all distributional variations in $S$ for which conditions (A1) remain satisfied.

The sufficiency direction in part 2 follows from theorem 3.3 in the manner of part 1, above, noting that conditions (A2) are in strict inequality form, and hence hold over a variational neighborhood of $S$ if they hold at $S$. The 
necessity direction follows by noting that $p^{B}$ and $i$ are not independent of all $\varepsilon$-distributional variations in $\omega$ for any $\varepsilon>0$ in any of the other liquidity preference regimes with $i>0$.

Part 3 similarly follows by examining the form of the solutions under the hypotheses of theorems 3.3 through 3.8 and their symmetrical counterparts. Q.E.D.

Proof of theorem 4.3. By theorem 3.2, given $N \omega^{1}>0, N \omega^{2}=0$, and $T N S>0$, a necessary and sufficient condition for the existence of a zero interest rate equilibrium for $e$ is the existence of values $\bar{B}_{1}$ and $\bar{B}_{2}$ satisfying $N_{1} \bar{B}_{1}+N_{2} \bar{B}_{2}$ $=\bar{B}$ and

$$
\begin{aligned}
& \frac{(1+\alpha+\beta) \bar{B}_{k}+S_{k}}{N S} \leqq\left[\frac{1-T}{T}\right] \frac{(\alpha+\beta) \omega_{k}^{1}}{N \omega^{1}}, \quad k=1,2, \\
& \frac{(1+\alpha+\beta) \bar{B}_{k}+(1+\alpha) S_{k}}{N S} \leqq \beta\left[\frac{1-T}{T}\right] \frac{\omega_{k}^{1}}{N \omega^{1}}, \quad k=1,2 .
\end{aligned}
$$

Clearly, condition (B12) holds whenever condition (B13) holds. When multiplied through by $N_{k}$ and aggregated over $k$, condition (B13) reduces to

$$
(\bar{B}+N S) T \leqq\left(\frac{\beta}{1+\alpha+\beta}\right) N S .
$$

Conversely, if (B14) holds, there exist values $\bar{B}_{1}^{*}$ and $\bar{B}_{2}^{*}$ satisfying $N_{1} \bar{B}_{1}^{*}$ $+N_{2} \bar{B}_{2}^{*}=\bar{B}$ together with condition (B13). Specifically, let

$$
\begin{aligned}
& \bar{B}_{1}^{*} \equiv\left[\bar{B}-N_{2} \bar{B}_{2}^{*}\right] / N_{1}, \\
& \bar{B}_{2}^{*} \equiv\left(\frac{1}{1+\alpha+\beta}\right)\left[\frac{\beta N S\lceil 1-T\rceil \omega_{2}^{1}}{T N \omega^{1}}-(1+\alpha) S_{2}\right] .
\end{aligned}
$$

Substituting (B16) into (B13) for $k=2$ yields identical left and right sides. Substituting (B15) into (B13) for $k=1$, the left-hand side becomes, after some reductions,

$$
\frac{(1+\alpha+\beta) \bar{B}+(1+\alpha) N S}{N_{1} N S}-\frac{\beta[1-T] N_{2} \omega_{2}^{1}}{N_{1} T N \omega^{1}}
$$

It can be shown that (B17) is less than or equal to the right-hand side of (B13) for $k=1$ if and only if (B14) holds. 
Conditions (B12) and (B13) are therefore equivalent to (B14). It follows that condition (B14) is necessary and sufficient for the existence of a zero interest rate equilibrium for $e$. Since each distinct pair of values $\left(\bar{B}_{1}, \bar{B}_{2}\right)$ yields a distinct zero interest rate equilibrium, infinitely many exist if (B14) holds with strict inequality and a unique one exists [corresponding to $\bar{B}_{1}^{*}$ and $\bar{B}_{2}^{*}$ in (B15) and (B16)] if (B14) holds with equality.

Finally, it can be verified from the form of the solution in theorem 3.2 that $M_{k}^{1}>M_{k}^{2}, k=1,2$, for any zero interest rate equilibrium for $e$.

Concerning part 2 , it follows from theorem 3.3 that a unique equilibrium exists for $e$ satisfying $i>0$ and $\left(M_{k}^{1}>0, M_{k}^{2}=0\right), k=1,2$, if and only if $\beta N S /(1$ $+\alpha+\beta)<(\bar{B}+N S) T$. Moreover, it can be verified that no other equilibria exist for $e$ with $i>0$, since $N \omega^{2}=0$ violates the basic necessary and sufficient conditions for existence in theorems 3.4 through 3.8 , and also in the symmetrical counterpart theorems $3.6^{*}$ through $3.8^{*}$.

Thus, parts 1 and 2 are established, and part 3 is then a direct corollary of theorem 4.2. Q.E.D.

Proof of theorem 4.4. If $T N S>0$, then, by theorem 3.2, a necessary condition for the existence of an equilibrium for $e$ with $i=0$ is the existence of values $\bar{B}_{1}$ and $\bar{B}_{2}$ satisfying $N_{1} \bar{B}_{1}+N_{2} \bar{B}_{2}=\bar{B}$ and

$$
\frac{(1+\alpha+\beta) \bar{B}_{k}+(1+\alpha) S_{k}}{\beta N S} \leqq \frac{1-T}{T} \frac{\omega_{k}^{2}}{N \omega^{2}} \leqq \frac{(1+\alpha+\beta)\left[-\bar{B}_{k}\right]-S_{k}}{N S}, \quad k=1,2,
$$

which implies

$$
(1+\alpha+\beta) S_{k} \leqq(1+\beta)(1+\alpha+\beta)\left[-\bar{B}_{k}\right], \quad k=1,2
$$

Multiplying through (B18) by $N_{k}$ and aggregating with respect to $k$ yields condition (6). If $T N S=0$, then, by theorem 3.2, existence with $i=0$ requires $T$ $=N S=0$ and $\bar{B}<0$, i.e. $0<(1+\beta)[-\bar{B}]$, so again condition (6) holds.

In similar fashion, it can be shown that the necessary conditions for existence given in theorems 3.3 through 3.8 and their symmetrical countcrparts imply the satisfaction of condition (6). Q.E.D.

Proof of theorem 4.5. Using theorems 3.2 through 3.8 , the following five assertions can be verified for $N S=T=\bar{B}=0$. (In each case, if an equilibrium exists, then infinitely many exist.)

1. (Theorem 3.2) There exists a zero interest rate equilibrium for $e$ if and only if

$$
(1+\alpha+\beta) b_{k} \leqq \min \left\{(\alpha+\beta) \omega_{k}^{1}-\omega_{k}^{2}, \beta\left[\omega_{k}^{1}+\omega_{k}^{2}\right]\right\}, \quad k=1,2,
$$


for some $b_{1}$ and $b_{2}$ in $R$ satisfying $N_{1} b_{1}+N_{2} b_{2}=0$. (Given any such values $b_{1}$ and $b_{2}$, any value $p^{B}>0$ yields an equilibrium price with corresponding bond values $\bar{B}_{1} \equiv b_{1} / p^{B}$ and $\bar{B}_{2} \equiv b^{2} / p^{B}$.)

2. (Theorem 3.5) There exists an equilibrium for $e$ with $\left(M_{k}^{1}=0, M_{k}^{2}>0\right), k$ $=1,2$, if and only if

$$
\begin{aligned}
& 0<(\alpha+\beta) N \omega^{1}<N \omega^{2}, \\
& \frac{\alpha}{1+\beta}\left(\frac{N \omega^{2}}{(\alpha+\beta) N \omega^{1}}\right) \omega_{1}^{1}<\omega_{1}^{2}, \\
& \frac{\alpha}{1+\beta}\left(\frac{N \omega^{2}}{(\alpha+\beta) N \omega^{1}}\right) \omega_{2}^{1}<\omega_{2}^{2} .
\end{aligned}
$$

3. (Theorem 3.7) There exists an equilibrium for $e$ with $\left(M_{1}^{1}=0, M_{1}^{2}>0\right)$ and $\left(M_{2}^{1}=0, M_{2}^{2}=0\right)$ if and only if

$$
\begin{aligned}
& 0<L<(1+\beta) N_{1} \omega_{1}^{2}, \\
& \alpha \omega_{2}^{1} \leqq(1+\beta) \omega_{2}^{2}, \\
& L \omega_{2}^{2} \leqq\left(\alpha N_{1} \omega_{1}^{2}\right) \omega_{2}^{1},
\end{aligned}
$$

where $L \equiv(1+\beta)(\alpha+\beta) N_{1} \omega_{1}^{1}+\beta(1+\alpha+\beta) N_{2} \omega_{2}^{1}$.

4. (Theorem 3.8) There exists an equilibrium for $e$ with $\left(M_{1}^{1}=0, M_{1}^{2}>0\right)$ and $\left(M_{2}^{1}>0, M_{2}^{2}=0\right)$ if and only if

$$
\begin{aligned}
& 0<(\alpha+\beta) N_{1} \omega_{1}^{1}+\beta N_{2} \omega_{2}<N_{1} \omega_{1}^{2}, \\
& (1+\beta) \omega_{2}^{2}<\alpha \omega_{2}^{1} .
\end{aligned}
$$

5. No equilibria exist for $e$ with $M_{k}^{2}=0, k=1,2$.

By symmetry, there are two more regions of existence corresponding to cases 3 and 4 (theorems $3.7^{*}$ and $3.8^{*}$ ) with the roles of agent types 1 and 2 interchanged.

It can then be shown that, for each fixed set of values for $N, \omega_{1}^{1}, \omega_{2}^{1}, \alpha$, and $\beta$ with $N \omega^{1}>0$, conditions 1 through 5 define a partition of $\left(\omega_{1}^{2}, \omega_{2}^{2}\right)$-space, as depicted in fig. 5. None of the conditions 1 through 5 is satisfied if $N \omega^{1}$ $=0$, an intuitively predictable result; for both agent types would need to borrow (sell bonds) in period 1 in this case, which is incompatible with $\bar{B}$ $=0$. Q.E.D. 
Proof of theorem 4.6. The proof of theorem 4.6 is analogous to the proof of theorem 4.5, consisting of a detailed examination of the necessary and sufficient conditions defining the boundaries of the liquidity preference regions in theorems 3.2 through 3.8 and their symmetrical counterparts under the special assumptions $N_{1}=N_{2}, \omega_{1}^{1}=\omega_{2}^{1} \equiv \bar{\omega}, \omega_{1}^{2}=\omega_{2}^{2}=1-\bar{\omega}$, and $\bar{B}$ $=S_{2}=0$. Q.E.D.

\section{References}

Balasko, Y. and K. Shell, 1981a, The overlapping-generations model, II: The case of pure exchange with money, Journal of Economic Theory 24, 112-142.

Balasko, Y. and K. Shell, 1981b, The overlapping-generations model III: The case of $\log$-lincar utility functions, Journal of Economic Theory 24, 143-152.

Brock, W. and J. Scheinkman, 1980, Some remarks on monetary policy in an overlapping generations model, in: J. Kareken and N. Wallace, eds., Models of monetary economics (Federal Reserve Bank of Minneapolis, Minneapolis) 211-232.

Cass, D. and K. Shell, 1980, In defense of a basic approach, in: J. Kareken and N. Wallace, eds., Models of monetary economics (Federal Reserve Bank of Minneapolis, Minneapolis) 251260.

Green, H.A.J., 1977, Aggregation problems of macroeconomics, in: G.C. Harcourt, ed., The Microeconomic Foundations of Macroeconomics (Westview Press, Colorado) 179-194.

Lucas, R., 1980, Equilibrium in a pure currency economy, in: J. Kareken and N. Wallace, eds., Models of monetary economics (Federal Reserve Bank of Minneapolis, Minneapolis) 131145.

Lucas, R. and T. Sargent, 1979, After Keynesian economics, Quarterly Review, Spring.

Martins, M., 1980, A nominal theory of the nominal rate of interest and the price level, Journal of Political Economy 88, 174-185.

Nikaido, H., 1968, Convex structures and economic theory (Academic Press, New York).

Sargent, T. and N. Wallace, 1976, Rational expectations and the theory of monetary policy, Journal of Monetary Economics 2, 169-183.

Wallace, N., 1980a, The overlapping generations model of fiat money, in: J. Kareken and N. Wallace, eds., Models of monetary economics (Federal Reserve Bank of Minneapolis, Minneapolis) 49-82.

Wallace, N., 1980b, Existence of optimal fixed-supply monetary equilibrium in simple, monotone excess-demand overlapping generations models, unpublished manuscript, April. 